\title{
Platelet-Specific Deletion of Cyclooxygenase-1 Ameliorates Dextran Sulfate Sodium-Induced Colitis in Mice ${ }^{\mathbb{S}}$
}

\begin{abstract}
Angela Sacco, ${ }^{1}$ Annalisa Bruno, ${ }^{1}$ Annalisa Contursi, ${ }^{2}$ Melania Dovizio, ${ }^{2}$ Stefania Tacconelli, $^{3}$ Emanuela Ricciotti, Paloma Guillem-Llobat, Tania Salvatore, Luigia Di Francesco, Rosa Fullone, Patrizia Ballerini, Vincenzo Arena, Sara Alberti, Guizhu Liu, Yanjun Gong, Alessandro Sgambato, Carlo Patrono, Garret A. FitzGerald, Ying Yu, and Paola Patrignani

Department of Neuroscience, Imaging, and Clinical Sciences and Center for Research on Aging and Translational Medicine, "G. d'Annunzio" University School of Medicine, Chieti, Italy (A.Sa., A.B., A.C., M.D., S.T., P.G.-L., T.S., L.D.F., R.F., P.B., S.A., P.P.); Department of Systems Pharmacology and Translational Therapeutics and Institute for Translational Medicine and Therapeutics, Perelman School of Medicine, University of Pennsylvania, Philadelphia, Pennsylvania (E.R., G.A.F.); Departments of General Pathology N.A., A.Sg.) and Pharmacology (C.P.), Catholic University School of Medicine, Rome, Italy; Institute for Nutritional Sciences, Shanghai Institutes for Biological Sciences, Graduate School of the Chinese Academy of Sciences, Chinese Academy of Sciences, Shanghai, China (G.L., Y.G.); and Department of Pharmacology, School of Basic Medical Sciences, Tianjin Medical University, Tianjin, China (Y.Y.)
\end{abstract}

Received April 23, 2019; accepted June 24, 2019

\begin{abstract}
Inflammatory bowel disease (IBD) is associated with an increased risk for thromboembolism, platelet activation, and abnormalities in platelet number and size. In colitis, platelets can extravasate into the colonic interstitium. We generated a mouse with a specific deletion of cyclooxygenase (COX)-1 in megakaryocytes/platelets [(COX-1 conditional knockout (cKO)] to clarify the role of platelet activation in the development of inflammation and fibrosis in dextran sodium sulfate (DSS)induced colitis. The disease activity index was assessed, and colonic specimens were evaluated for histologic features of epithelial barrier damage, inflammation, and fibrosis. Cocultures of platelets and myofibroblasts were performed. We found that the specific deletion of COX-1 in platelets, which recapitulated the human pharmacodynamics of low-dose aspirin, that is, suppression of platelet thromboxane $(\mathrm{TX}) \mathrm{A}_{2}$ production associated with substantial sparing of the systemic production of prostacyclin, resulted in milder symptoms of colitis, in the acute phase, and almost complete recovery from the disease after DSS withdrawal. Reduced colonic accumulation of macrophages and myofibroblasts and collagen deposition was found. Platelet-derived $\mathrm{TXA}_{2}$ enhanced the ability of myofibroblasts to
\end{abstract}

proliferate and migrate in vitro, and these effects were prevented by platelet COX-1 inhibition or antagonism of the $\mathrm{TXA}_{2}$ receptor. Our findings allow a significant advance in the knowledge of the role of platelet-derived $\mathrm{TXA}_{2}$ in the development of colitis and fibrosis in response to intestinal damage and provide the rationale to investigate the potential efficacy of the antiplatelet agent low-dose aspirin in limiting the inflammatory response and fibrosis associated with IBD.

\section{SIGNIFICANCE STATEMENT}

Inflammatory bowel disease (IBD) is characterized by the development of a chronic inflammatory response, which can lead to intestinal fibrosis for which currently there is no medical treatment. Through the generation of a mouse with specific deletion of cyclooxygenase-1 in megakaryocytes/platelets, which recapitulates the human pharmacodynamics of lowdose aspirin, we demonstrate the important role of plateletderived thromboxane $A_{2}$ in the development of experimental colitis and fibrosis, thus providing the rationale to investigate the potential efficacy of low-dose aspirin in limiting the inflammation and tissue damage associated with IBD.

\section{Introduction}

Inflammatory bowel diseases (IBDs), including ulcerative colitis (UC) and Crohn's disease (CD), are complex and

This work was supported by Associazione Italiana per la Ricerca sul Cancro (AIRC) [IG 2017-ID. 20365 Project; Principal Investigator P.P.] and by Ministero dell' Istruzione, dell' Università e della Ricerca (MIUR) [Fondi per la Ricerca Scientifica di Ateneo, (ex 60\%)] to P.P.; Angela Sacco was supported by a fellowship from Società Italiana di Farmacologia-Merck Sharp Dohme (SIF-MSD). In addition, this work was conducted on behalf of the Aspirin for Cancer Prevention Group, Wolfson Institute of Preventive Medicine, Queen Mary School of Medicine and Dentistry, University of London (London, UK).

${ }^{1}$ A.Sa. and A.B. contributed equally, as first coauthors.

${ }^{2}$ A.C. and M.D. contributed equally, as second coauthors.

${ }^{3}$ S.T. contributed equally, as second coauthor.

https://doi.org/10.1124/jpet.119.259382.

S This article has supplemental material available at jpet.aspetjournals.org. multifactorial diseases of unknown etiology (Ananthakrishnan, 2015). CD and UC are chronic remittent or progressive inflammatory conditions that may affect the entire gastrointestinal tract or the colorectal mucosa, respectively, and are associated with increased risk for colon cancer (Choi et al., 2017).

The efficacy of agents that target inflammatory/immune pathways (Shah and Mayer, 2010) supports the involvement of a dysregulated response to damage/dysfunction of the epithelial monolayer lining the large intestine, in the development of IBD (Abraham and Cho, 2009; Ananthakrishnan, 2015). However, this therapeutic strategy is limited by intersubject variability in the response and the risk of infection or its reactivation (Abraham and Cho, 2009; Shah and Mayer, 
2010). Impaired tissue repair in IBD may lead to intestinal fibrosis for which currently there is no medical treatment (Rieder and Fiocchi, 2008). Therefore, the development of therapies that are more efficacious, safer, and possibly tailored to the individual characteristics of the patient with IBD represents a current challenge.

IBD is associated with increased risk for thromboembolism, platelet activation, and abnormalities in platelet number and size (Kapsoritakis et al., 2001; Giannotta et al., 2015). Besides their role in hemostasis, platelets may contribute to the development of inflammatory diseases, through their ability to orchestrate the activation of vascular, immune, and inflammatory cells by the release of a wide array of bioactive molecules, including thromboxane (TX)A $\mathrm{A}_{2}$ (Gawaz et al., 2005; Dixon et al., 2006; Davì and Patrono, 2007; Dovizio et al., 2014). In both human and murine platelets, $\mathrm{TXA}_{2}$ is the major product of arachidonic acid metabolism via the activity of cyclooxygenase (COX)-1 (Simmons et al., 2004; Smyth et al., 2009; Ricciotti and FitzGerald, 2011). Intestinal epithelial and inflammatory cells may also generate $\mathrm{TXA}_{2}$, although at considerably lower levels (Simmons et al., 2004; Smyth et al., 2009; Ricciotti and FitzGerald, 2011). The actions of $\mathrm{TXA}_{2}$ are mediated through the activation of receptors designated TX-prostanoid receptors (TPs) belonging to the superfamily of $\mathrm{G}$ protein-coupled receptors (Simmons et al., 2004). $\mathrm{TXA}_{2}$ is a potent vasoconstrictor and induces platelet aggregation (Simmons et al., 2004; Davì and Patrono, 2007; Ricciotti and FitzGerald, 2011). It also enhances lymphocyte and macrophage functions (Leung and Mihich, 1980; Ceuppens et al., 1985; Thomas et al., 2003) and stimulates the biosynthesis of extracellular matrix proteins (Bruggeman et al., 1991). In IBD patients, platelets have been observed to infiltrate the colon interstitium and move into the gut lumen (Weissmüller et al., 2008). Enhanced biosynthesis of $\mathrm{TXB}_{2}$ (the stable hydrolysis product of $\mathrm{TXA}_{2}$ ) by rectal mucosa from patients with CD has been detected even in the absence of inflammation (Hawkey et al., 1983). Elevated $\mathrm{TXA}_{2}$ levels are associated with necrosis and ulceration of the stomach (Whittle et al., 1981). The administration of the TP antagonist, vapiprost, to mice alleviated dextran sulfate sodium (DSS)-induced colitis (Carter et al., 2011). However, these studies did not identify the cellular source(s) of $\mathrm{TXA}_{2}$ involved in the development of colitis. Also, the possible role of $\mathrm{TXA}_{2}$ in the persistent and unresolved mucosal inflammation leading to intestinal fibrosis, frequently associated with IBD (Rieder and Fiocchi, 2008), remains to be investigated.

In the present study, we tested the hypothesis that plateletderived $\mathrm{TXA}_{2}$ may play a role in the acute phase and recovery phase of colitis induced in mice by DSS administration, which causes damage to the epithelial monolayer lining of the large intestine resembling human UC (Chassaing et al., 2014). To address this objective, we generated a mouse with specific deletion of COX-1 [(COX-1 conditional knockout (cKO)] in megakaryocytes/platelets. DSS administration to COX-1 cKO mice induced milder colitis symptoms, lesser histologic lesions, and inflammatory markers in the acute phase and almost complete recovery from the disease after DSS withdrawal (chronic phase). Reduced systemic $\mathrm{TXA}_{2}$ biosynthesis in COX-1 cKO mice was associated with lower colonic collagen deposition and myofibroblast number in the chronic phase of colitis. In vitro studies also revealed that platelet-derived $\mathrm{TXA}_{2}$ plays a crucial role in the promotion of fibrosis via the induction of phenotypic and functional changes in myofibroblasts. Our results suggest novel therapeutic strategies in IBD.

\section{Materials and Methods}

Generation and Characterization of Mice with a Specific Deletion of COX-1 in Megakaryocytes/Platelets. We generated the floxed Ptgs1 (COX-1) mouse, where exons 6 and 7 were flanked by loxP sites (Supplemental Fig. 1A), using as genome-editing tool the transcription activator-like effector nucleases, which significantly increase the efficiency of genomic modification (Joung and Sander, 2013). These mice were crossed with platelet factor 4 (Pf4)-Cre transgenic C57BL/6 mice [purchased from Jackson Laboratories (Bar Harbor, ME)] that express a codon-improved Cre recombinase (iCre) under the control of the mouse Pf4 promoter, leading to the Cre recombinase expression in the majority of megakaryocytes. The Cremediated excision of the COX-1 allele and the original COX-1flox allele was confirmed by specific polymerase chain reaction (PCR)based genotyping assays of tail biopsies (Supplemental Fig. 1B). For genotyping of mice, tail genomic DNA was extracted by using Wizard Genomic DNA Purification Kit (Promega, Milan, Italy). Then the DNA lysates were subjected to PCR analysis with the following primers: $\operatorname{ptgs}^{f l x}$ (forward, 5'-ACTTCTATGCTGGTGGACTATGG-3'; reverse, 5'-TCTGGTGTGATGCCTGTGCTAT-3', wild-type 599-bp product, floxed 671-bp product) and PF4 Cre (transgene forward, $5^{\prime}$-CCAAGT CCTACTGTTTCTCACTC-3'; transgene reverse, 5'-TGCACAGTC AGCAGGTT-3'; internal positive control forward, 5'-CTAGGCCAC AGAATTGAAAGATCT-3'; internal positive control reverse, 5'-GTA GGTGGAAATTCTAGCATCATCC-3', transgene 420-bp product, internal positive control 324-bp product).

The specific deletion of platelet COX-1 was verified by assessing the COX-1 levels by Western blot (Patrignani et al., 2017) in isolated platelets, peritoneal macrophages, and different tissues/organs (colon, intestinal mucosa, kidney, and uterine tubes), collected from wild-type and COX-1 cKO mice, as reported in Supplemental Material. Platelet COX-1 capacity to generate $\mathrm{TXB}_{2}$ was evaluated in whole blood, collected from the submandibular vein of the two groups of mice, allowed to clot at $37^{\circ} \mathrm{C}$ for 1 hour (Patrono et al., 1980). Bleeding time was assessed, as previously reported (Yu et al., 2005).

DSS-Induced Colitis in Mice and Sample Size Calculation. The animal experiments were performed in accordance with the European Communities Council Directive of September 22, 2010 (2010/63/EU) and the National Ethical Committee. The study was approved by the Italian Ministry of Health, authorization n. 956/2016PR and 115/2019-PR. The animals were housed in cages up to five mice each and acclimated under conditions of controlled temperature $(20 \pm$ $\left.2{ }^{\circ} \mathrm{C}\right)$, humidity (55\% $\left.\pm 10 \%\right)$, and lighting (7:00 AM to 7:00 PM). For all of the experiments, mice were housed under specific pathogen-free conditions and allowed free access to food and water, and the animals'

ABBREVIATIONS: $\alpha$-SMA, $\alpha$-smooth muscle actin; AUC, area under the curve; BSA, bovine serum albumin; CD, Crohn's disease; cKO conditional knockout COX, cyclooxygenase; DAI, disease activity index; DSS, dextran sulfate sodium; HPGDS, hematopoietic PGD synthase; IBD, inflammatory bowel disease; IL, interleukin; LPGDS, lipocalin-type prostaglandin D synthase; mPGES1, microsomal PGE synthase 1; PCR, polymerase chain reaction; Pf4, platelet factor 4; PG, prostaglandin; PGD-M, 11,15-dioxo-9 $\alpha$-hydroxy-2,3,4,5-tetranorprostan-1,20-dioic acid; PGE-M, 7-hydroxy-5,11-diketotetranorprostane-1,16-dioic acid; PGI-M, 2,3-dinor 6-keto-PGF ${ }_{1 \alpha}$; TGF, transforming growth factor; TP, TXprostanoid receptor; TX, thromboxane; TX-M, 2,3-dinor $\mathrm{TXB}_{2}$; UC, ulcerative colitis. 
health status was monitored daily. All efforts were made to minimize animal suffering and to reduce the number of animals used. The investigator assessing phenotype was unaware of the genotype throughout these experiments.

We used the colitis model that employs $2 \%$ DSS (Okayasu et al., 1990; Cooper et al., 1993) (36-50 kDa) (D.B.A. Italia s.r.l, Milan, Italy) dissolved in drinking water, administered to female C57BL/6 wildtype mice (8-10 weeks old, $20-25$ g body weight) for 5 days (acute phase), followed by the tap water for an additional 16 days (chronic phase) (Supplemental Fig. 2). The model was developed in 11 wildtype mice, and the primary endpoint was the measurement of the area under the curve (AUC; curve of scores vs. time) from day 0 to 21 of the disease activity index (DAI) (Okayasu et al., 1990; Cooper et al., 1993), which is calculated as the sum of blood in stool (EMO score) and stool consistency (STOOL score) (Supplemental Table 1), as reported in detail in Supplemental Material. This number of mice was selected to have at least five mice completing the 21-day study. We did not find any animal loss for mortality in wild-type mice treated with DSS, and the AUC of DAI values showed a coefficient of variation of $10 \%$; thus, five COX-1 cKO mice were treated with DSS. This sample size had a $95 \%$ power to detect a difference between means of the AUC of DAI values of at least 13.73 with a significance level $(\alpha)$ of 0.05 (two-tailed).

Urinary Levels of Enzymatic Metabolites of Prostanoids. In wild-type and COX-1 cKO mice, 24-hour urine collections were performed with the use of metabolic cages. Systemic production of prostaglandin $(\mathrm{PG}) \mathrm{E}_{2}, \mathrm{PGD}_{2}, \mathrm{PGI}_{2}$, and $\mathrm{TXA}_{2}$ was determined by quantifying the urinary levels of their major enzymatic metabolites: 7-hydroxy-5,11-diketotetranorprostane-1,16-dioic acid (PGE-M), 11,15-dioxo- $9 \alpha$-hydroxy-2,3,4,5-tetranorprostan-1,20-dioic acid (tetranor PGD-M), 2,3-dinor 6-keto-PGF ${ }_{1 \alpha}$ (PGI-M), and 2,3-dinor $\mathrm{TXB}_{2}$ (TX-M), respectively, by liquid chromatography/tandem mass spectrometry, as previously described (FitzGerald et al., 1983; Song et al., 2007, 2008; Wang and DuBois, 2013). Data were normalized for urinary creatinine (Oxford Biomedical Research, Rochester Hills, MI).

Assessment of Prostanoid Levels in Colon Sample. Colon samples, collected at the sacrifice, were homogenized at $4^{\circ} \mathrm{C}$ in $300 \mu \mathrm{l}$ PBS supplemented with Complete protease inhibitor mixture (Roche, Milan, Italy) and $20 \mu \mathrm{M}$ indomethacin (Sigma-Aldrich, Milan, Italy) and sonicated for 30 seconds. The homogenates were first centrifuged at $8000 \mathrm{rpm}$ for 10 minutes, and the supernatant was used for protein determination and for prostanoid measurements (Boulet et al., 2004). The 6-keto-PGF $1 \alpha, \mathrm{PGE}_{2}$, and $\mathrm{TXB}_{2}$ levels were measured by previously described immunoassay techniques (Di Francesco et al., 2009). PGD $_{2}$ levels were measured by enzyme immunoassay, according to the protocol of the manufacturer (Cayman Chemical, Ann Arbor, MI). Data were normalized for protein concentration.

Assessment of Cytokine and Protein Levels in Colon Samples. Colon samples collected at the sacrifice were suspended in $150 \mu$ l lysis buffer (Din et al., 2012), underwent three cycles of freeze (5 minutes)-thawing, and were homogenized in ice. Then homogenates were put on ice for 30 minutes. Cell debris was removed by centrifuging (13,000 rpm, 5 minutes at $4^{\circ} \mathrm{C}$ ) (Din et al., 2012). Samples were stored at $-80^{\circ} \mathrm{C}$ until the analyses. Interleukin (IL)-10, IL-6, and transforming growth factor (TGF)- $\beta 1$ levels were determined by ELISA (Diaclone SAS, Besancon Cedex, France), according to the protocol of the manufacturer, and data were normalized for protein concentration. The expression levels of COX-1, COX-2, microsomal PGE synthase1 (mPGES1), hematopoietic PGD synthase (HPGDS), lipocalin-type prostaglandin D synthase (LPGDS), and $\beta$-actin were assessed by Western blot (Patrignani et al., 2017), using the following antibodies: anti-COX-1 (1:1000, catalogue 160109; Cayman Chemical), anti-COX-2 (1:1000, catalogue 160126; Cayman Chemical), antimPGES1 (1:1000, catalogue 160140; Cayman Chemical), anti-HPGDS (1:200, catalogue 10004345; Cayman Chemical), anti-LPGDS (1:200, catalogue 10004344; Cayman Chemical), and $\beta$-actin (1:1000, catalogue sc-1615; Santa Cruz Biotechnology) as loading control. The quantification of the optical density of different specific bands was calculated using Alliance $1 \mathrm{D}$ software (UVITEC, Cambridge, UK) and normalized to the optical density of $\beta$-actin.

Histopathological Analysis of Colon Tissue Samples. Distal colon was fixed in buffered 10\% formalin for 12-24 hours, dehydrated, and embedded in paraffin for histologic analysis. Sections of $5 \mu \mathrm{m}$ were obtained from paraffin blocks and were stained with H\&E (Bio-Optica, Milan, Italy) following the standard protocol to assess the ulcer score, the ulcer area score, the inflammatory score, the area of the inflammatory score, the total histopathological score (Supplemental Table 2), and the percentage of necrosis area. Necrosis areas were analyzed using Image $J$ software $(\mathrm{NIH})$. For each sample, 10 representative images were taken with a $10 \times$ objective. The percentage of loss intestinal architecture area/total area was measured. To highlight the degree of collagen depositions, the colonic sections were also stained using Masson's Trichrome Staining (Bio-Optica), according to the protocol of the manufacturer. Briefly, coverslips were mounted with Balsamo Eukitt (Bio-Optica) and evaluated under a light microscope. Image processing was performed using Image $\mathrm{J}$ software (NIH). Positive areas of collagen fibers were measured and expressed as percentage of total area.

Immunofluorescence Analysis of Colon Tissue Samples by Confocal Microscopy. Colon tissue sections were subjected to antigen retrieval by boiling the slides in DAKO target retrieval solution (DAKO, Santa Clara, CA) for 30 minutes. Sections were incubated in this solution for 20 minutes at room temperature and were sequentially incubated in protein-blocking solution [PBS with $3 \%$ bovine serum albumin (BSA)] for 30 minutes at room temperature. Primary antibodies anti-vimentin (1:100, catalogue ab188499; Abcam), anti- $\alpha$-smooth muscle actin ( $\alpha$-SMA, 1:100, catalogue sc32251; Santa Cruz Biotechnology), anti-integrin $\beta 3$ (1:100, CD61, catalogue sc-6627; Santa Cruz Biotechnology), anti-PECAM-1 (CD31, 1:100, catalogue sc-1506; Santa Cruz Biotechnology), and anti-F4/80 (1:100, catalogue sc-25830; Santa Cruz Biotechnology) were incubated with sections overnight at $4^{\circ} \mathrm{C}$. Sections were washed twice with PBS with $0.1 \%$ Tween (Sigma-Aldrich) and incubated with respective secondary antibodies. Then sections were stained with 1,5-bis \{[ 2-(dimethylamino) ethyl] amino\}-4,8-dihydroxyanthracene-9,10-dione (Biostatus, Leicestershire, UK) for the identification of nuclei. All sections were analyzed by using ZEISS LSM 510 META microscope.

Cultures of Human Intestinal Myofibroblasts and Isolated Human Platelets. The human intestinal myofibroblast cells used at passage levels 4-7, purchased from Clonetics (Lonza, Walkersville, MD), and derived from a male donor were cultured in smooth muscle medium-2 containing 5\% FBS, $1 \%$ gentamicin/amphotericin, $1 \%$ epidermal growth factor, $2 \%$ recombinant human basic fibroblast growth factor, and $1 \%$ insulin. In all experiments, $9 \times 10^{4}$ cells were seeded in a six-multiwell plate containing $3 \mathrm{ml}$ Dulbecco's modified Eagle's medium (Invitrogen, Milan, Italy) supplemented with 5\% FBS and $1 \%$ penicillin-streptomycin for 48 hours.

Human washed platelets were isolated from the concentrated buffy coat obtained by the Transfusion Centre (Renzetti Hospital of Lanciano, Italy) from healthy volunteers after signing informed consent, as previously described (Guillem-Llobat et al., 2016). In some experiments, platelets were pretreated with aspirin $(100 \mu \mathrm{M})$ for 30 minutes at room temperature to suppress platelet COX-1 activity. Washed platelets were cultured alone or with myofibroblasts for 24 or 48 hours. In some experiments, a highly selective TP antagonist, SQ 29,548 (10 $\mu \mathrm{M}$; Cayman Chemical) or U46619 (250 nM), a TXA 2 mimetic (Sigma-Aldrich), and/or TGF- $\beta 1$ $(0.33 \mathrm{ng} / \mathrm{ml}$; Promokine, Heidelberg, Germany) were used. In cell culture media, the levels of $\mathrm{TXB}_{2}$ were measured by a previously described immunoassay technique (Guillem-Llobat et al., 2016 ), and those of TGF- $\beta 1$ released in the medium were determined by ELISA (R\&D Systems, Minneapolis, MN). In myofibroblasts, the mRNA levels of $\alpha$-SMA, RhoA, vimentin, and fibronectin were assessed by quantitative real-time PCR, as reported in Supplemental Material. 
Immunofluorescence Analysis of $\alpha$-SMA in Myofibroblasts. Myofibroblasts were cultured alone or with platelets for 24 hours; then the cells were washed twice with PBS and fixed in cold acetone/ methanol solution (40:60) for 20 minutes at room temperature. Samples were blocked with a filtrated solution of 3\% BSA (SigmaAldrich) in PBS for 30 minutes at room temperature and incubated overnight at $4^{\circ} \mathrm{C}$ with an anti- $\alpha$-SMA monoclonal antibody (1:100, catalogue sc-32251; Santa Cruz Biotechnology). Samples were then washed three times with PBS and incubated with secondary antibodies. Cells were washed three times with PBS and incubated with 1,5-bis\{[ 2-(dimethylamino) ethyl] amino\}-4,8-dihydroxyanthracene9,10-dione as a nuclear marker (1:1000, DR50200; Biostatus) for 5 minutes. Confocal images were obtained using a Zeiss LSM 510 meta microscope (Carl Zeiss)

In Vitro Migration and Proliferation Assays. Cell migration of myofibroblasts cultured alone or with platelets was evaluated by scrape-wounding assay, as previously described (Terzuoli et al., 2018). Briefly, after 24 hours of platelet-myofibroblast cocultures, platelets were washed away. Cells, seeded on a six-multiwell dish, were scraped using a $200 \mu \mathrm{l}$ pipette tip, simulating a wound, incubated in Dulbecco's modified Eagle's medium, $0.75 \%$ BSA, and monitored periodically by light microscope up to 18 hours. Cell proliferation was evaluated by Click-iT EdU Alexa Fluor 488 cell proliferation assay kit (Invitrogen), according to the manufacturer's instructions.

Statistical Analysis. All values are reported as mean \pm S.D., unless otherwise stated. Statistical analysis among groups was determined by Student's $t$ test or one-way ANOVA, followed by Tukey's multiple comparisons test, using GraphPad Prism Software (version 7.00 for Windows; GraphPad, San Diego, CA). Values of $P<$ 0.05 were considered statistically significant. The DAI, STOOL, and EMO scores were also evaluated by assessing the AUC from days 0 to 21 (GraphPad Prism Software). The sample size determination was performed using GraphPad StatMate Software (GraphPad).

\section{Results}

Generation and Characterization of Mice with a Specific Deletion of COX-1 in Megakaryocytes/Platelets. We generated platelet COX-1 cKO mice using the Cre-loxP system, as a genetic tool to control site-specific recombination events in genomic DNA (Supplemental Fig. 1, A and B) (Ray et al., 2000). In the COX-1 cKO mice, COX-1 was not detectable in platelets, whereas it was present in peritoneal macrophages, colon, intestinal mucosa, kidney, and uterine tubes, as assessed by Western blot (Supplemental Fig. 1, C-E) (Patrignani et al., 2017). Tail vein bleeding time values were significantly $(P<0.01)$ higher in COX-1 cKO mice than in wild-type littermates (Supplemental Fig. 1F), and platelet COX-1 activity, as reflected by serum $\mathrm{TXB}_{2}$ levels (Patrono et al., 1980), was significantly $(P<0.01)$ reduced by $98.3 \% \pm 1.4 \%$ (Supplemental Fig. $1 \mathrm{G}$ ).

Effects of Platelet COX-1 Deletion on the Symptoms of DSS-Induced Colitis. The treatment of wild-type C57BL/6 female mice with DSS $(2 \%)$ for 5 days induced a time-dependent appearance of clinical symptoms of colitis, such as fecal bleeding and reduced stool consistency, with a maximal DAI score on day 5 that remained stable up to day 10, that is, 5 days after DSS withdrawal (Fig. 1). After that, the DAI score decreased but was still elevated versus baseline values (Fig. 1A). However, on day 13, visible fecal blood could no longer be detected (Fig. 1B), whereas the stool consistency remained loose up to day 21 (Fig. 1C). There was no mortality among the DSS-treated mice.

During the 5-day treatment with DSS, rectal bleeding (Fig. 1B), stool consistency (Fig. 1C), and their sum (DAI score) (Fig. 1A) did not differ between COX-1 cKO and

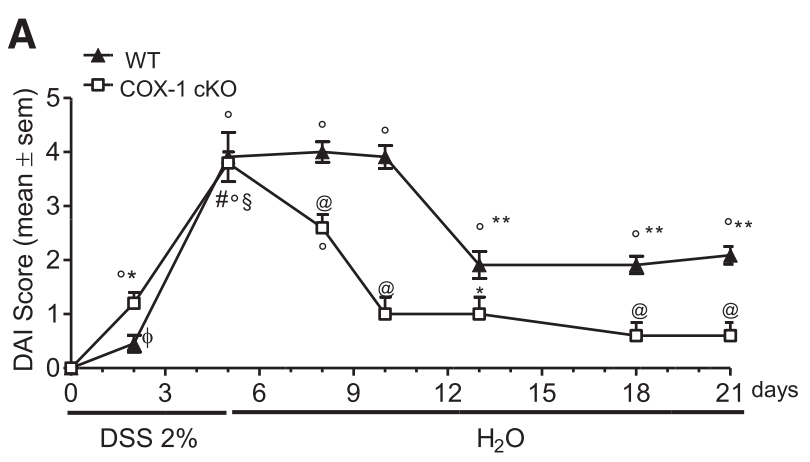

B
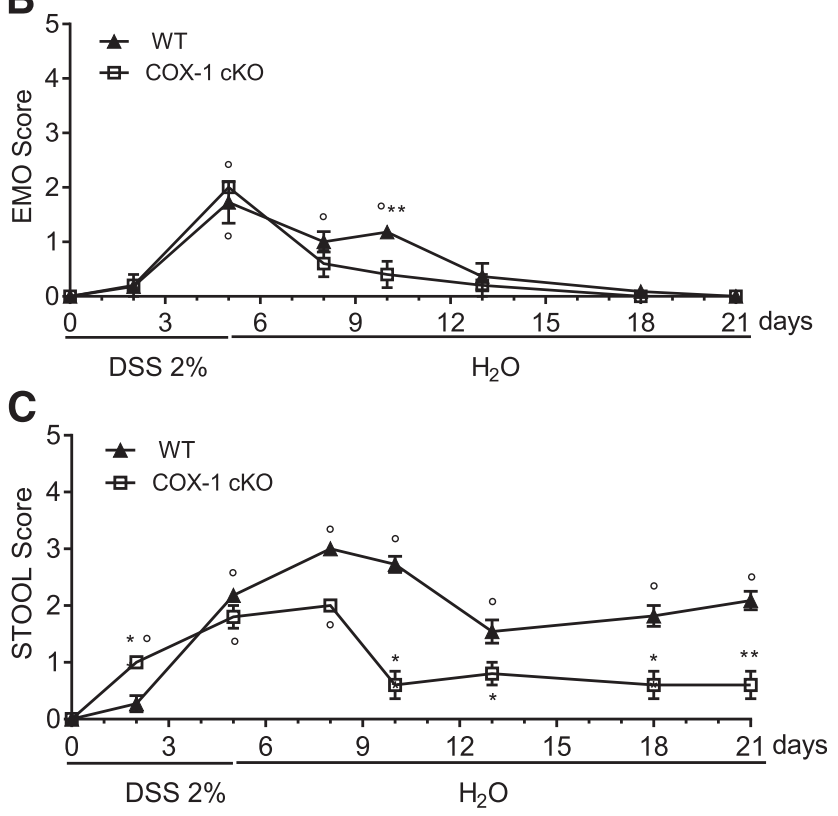

Fig. 1. Effects of platelet COX-1 deletion on the symptoms of DSSinduced colitis. Colitis was induced in C57BL/6 wild-type (WT) mice (female, 8-10 weeks old, $n=11$ ) and COX-1 cKO mice (female, 8-10 weeks old, $n=5$ ) by the administration of $2 \%$ DSS for 5 days (acute phase), followed by administration of tap water for further 16 days (chronic phase). The DAI score (A), EMO score (B), and STOOL score (C) were calculated at different time points, and data are reported as mean \pm S.E.M. (A) ${ }^{\mathrm{O}} P<0.01$ vs. baseline, day 0 , and ${ }^{\Phi} P<0.05$ vs. baseline, day 0 ; ${ }^{*} P<0.01$ vs. day 5,8 , and 10 of WT; $\S P<0.05$ vs. day 8 of COX-1 cKO; ${ }^{\#} P<0.01$ vs. days 10,13 , and 21 of COX- 1 cKO by Tukey's multiple comparisons test; $* P<0.05$ and ${ }^{\circledR} P<0.01$ vs. WT at the same time points by Student's $t$ test. (B) ${ }^{\mathrm{O}} P<0.01$ vs. baseline, day 0 , by Tukey's multiple comparisons test; $* * P<0.01$ vs. COX-1 cKO at the same time points by Student's $t$ test. (C) ${ }^{\circ} P<0.01$ vs. baseline, day 0 , by Tukey's multiple comparisons test; ${ }^{* * P}<0.01$ and $* P<0.05$ vs. WT at the same time points by Student's $t$ test.

wild-type mice. In contrast, after DSS withdrawal, a faster recovery of DAI score (both EMO and STOOL score) to baseline values was observed in the COX-1 cKO versus wildtype mice (Fig. 1). From day 10 to day 21, both EMO and STOOL scores were indistinguishable from baseline (Fig. 1, B and $\mathrm{C}$, respectively). The time-integrated disease activity (AUC) of DAI and STOOL scores estimated from day 0 to 21 resulted lower in COX-1 cKO mice than in wild-type mice (Supplemental Table 3). The AUC of EMO score was not significantly reduced in COX-1 cKO versus wild-type mice (Supplemental Table 3).

Effects of Platelet COX-1 Deletion on the Systemic Biosynthesis of Prostanoids in DSS-Induced Colitis. Urinary levels of the major enzymatic metabolites of $\mathrm{PGE}_{2}$, 
$\mathrm{PGD}_{2}, \mathrm{TXB}_{2}$, and $\mathrm{PGI}_{2}$ (prostacyclin), that are, PGE-M, PGDM, TX-M, and PGI-M, respectively, are reliable and noninvasive indexes of the systemic biosynthesis of the parent prostanoids (FitzGerald et al., 1983; Song et al., 2007, 2008; Wang and DuBois, 2013). As shown in Fig. 2, A and B, in wildtype mice urinary PGE-M and PGD-M were significantly increased on day 5 of DSS administration and returned to baseline values on day 21 . In contrast, the urinary levels of PGI-M and TX-M increased in a time-dependent fashion after DSS, and a significant change versus baseline was still detectable on day 21 (Fig. 2, C and D).

In COX-1 cKO mice, urinary TX-M levels were reduced by $60 \%$ at baseline, and this effect persisted after DSS administration (Fig. 2D). The specific deletion of platelet COX-1 was associated with lower urinary levels of PGE-M in the acute phase of colitis versus those detected in wild-type mice (Fig. 2A). It also restrained the enhanced biosynthesis of systemic $\mathrm{PGI}_{2}$ found in wild-type mice during the chronic phase of the disease (Fig. 2C). In contrast, the systemic biosynthesis of $\mathrm{PGD}_{2}$ was higher than in wild-type mice, after
DSS administration for 5 days (Fig. 2B). Altogether, these results show that deletion of platelet COX-1 restrains the enhanced systemic biosynthesis of $\mathrm{TXB}_{2}, \mathrm{PGE}_{2}$, and $\mathrm{PGI}_{2}$ occurring in DSS-induced colitis.

Effects of Platelet COX-1 Deletion on the Colonic Capacity to Produce Prostanoids in DSS-Induced Colitis. During the acute inflammatory response to DSS administration (day 5), enhanced capacity of the colonic tissue to form $\mathrm{PGE}_{2}, \mathrm{PGD}_{2}$, and 6-keto-PGF ${ }_{1 \alpha}$ (the stable hydrolysis product of $\mathrm{PGI}_{2}$ ), but not $\mathrm{TXB}_{2}$, was detected (Fig. 2, E-H). In COX-1 cKO mice, the colonic capacity to generate $\mathrm{PGE}_{2}$, $\mathrm{PGD}_{2}$, and 6-keto-PGF ${ }_{1 \alpha}$, but not $\mathrm{TXB}_{2}$, was significantly lower than that detected in wild-type mice (Fig. 2, E-H).

These data show that the specific deletion of COX-1 in platelets restrains the enhanced prostanoid biosynthesis induced in the colon by DSS administration. Interestingly, colonic $\mathrm{TXB}_{2}$ was not substantially affected by DSS challenge and platelet COX-1 deletion, thus suggesting that the enhanced systemic biosynthesis of $\mathrm{TXB}_{2}$ detected in colitis was from extracolonic origin mainly from platelets.
A

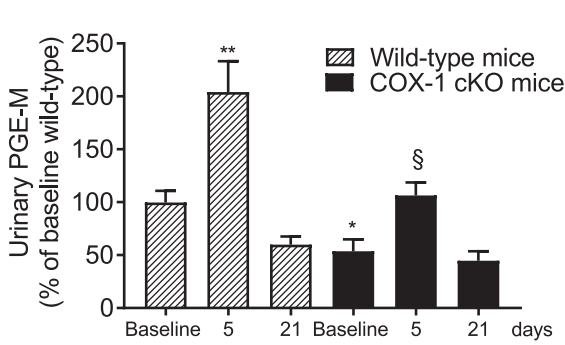

C

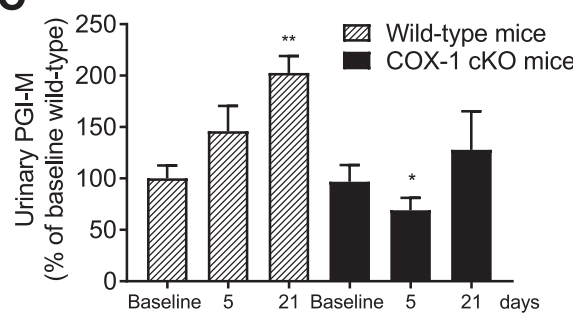

$\mathbf{E}$

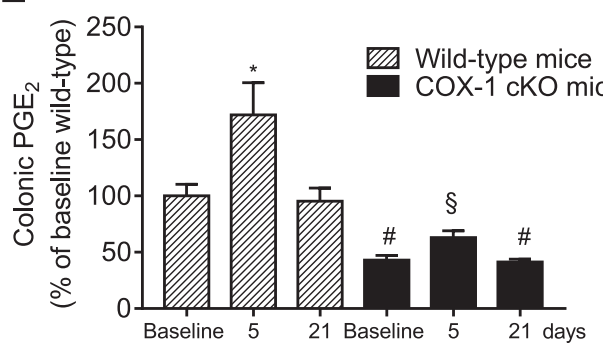

G

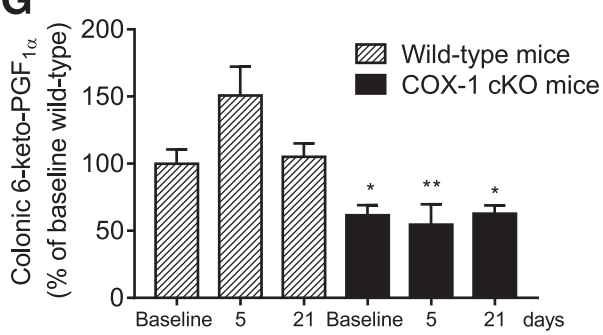

B

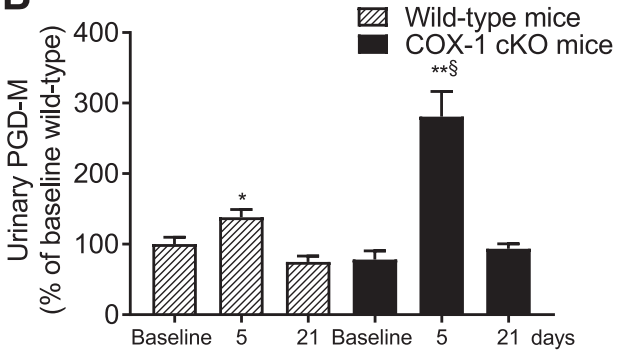

D

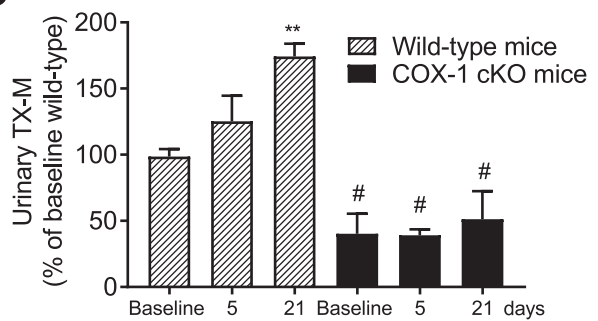

$\mathbf{F}$

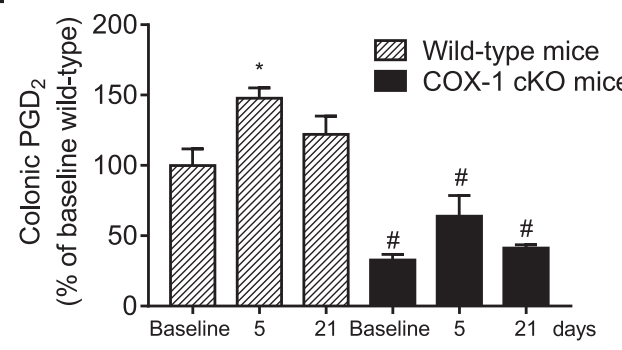

H

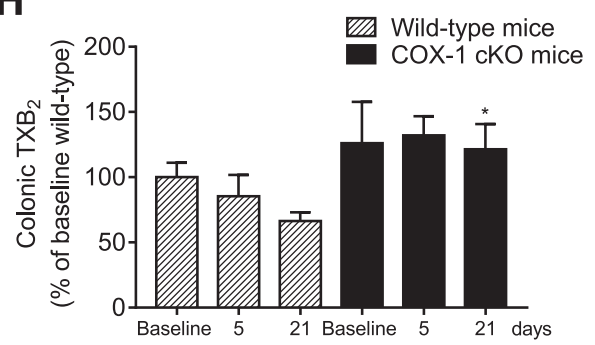

Fig. 2. Effects of platelet COX-1 deletion on systemic and colonic prostanoid biosynthesis in mice treated with DSS. (A-D) Twenty-four-hour urine samples were collected from wild-type and COX- 1 cKO mice on days 0,5 , and 21 to assess urinary levels of PGE-M, PGD-M, PGI-M, and TXM by LC/MS-MS; values were normalized for urinary creatinine; data are reported as percentage of baseline wild-type, mean \pm S.E.M. $(n=5-10)$. (A) $* * P<0.01$ vs. own baseline, day 0 , and day 21 by Tukey's multiple comparisons test; ${ }^{*} P<$ 0.05 vs. baseline of wild-type, ${ }^{\S} P<0.05$ vs. day 5 wild-type by Student's $t$ test. (B) $* P<0.05$ vs. own baseline, day $0, * * P<$ 0.01 vs. own baseline, day 0 , and day 21 by Tukey's multiple comparisons test; ${ }^{\circledR} P<$ 0.01 vs. day 5 of wild-type by Student's $t$ test. (C) **P $P<0.01$ vs. own baseline, day 0 by Tukey's multiple comparisons test; ${ }^{*} P<0.05$ vs. day 5 of wild-type by Student's $t$ test. (D) $* * P<0.01$ vs. own baseline, day 0 , by Tukey's multiple comparisons test; ${ }^{\#} P<0.01$ vs. the same time point of wild-type by Student's $t$ test. (E-H) The levels of $\mathrm{PGE}_{2}, \mathrm{PGD}_{2}, 6$-keto$\mathrm{PGF}_{1 \alpha}$, and $\mathrm{TXB}_{2}$ were assessed in colon samples $(n=4-8)$ collected on days 0,5 , and 21 from wild-type and COX-1 cKO mice, by specific immunoassays; values were normalized for tissue protein concentration and are reported as percentage of baseline of wild-type, mean \pm S.E.M. (E) $* P<0.05$ vs. own baseline, day 0 , by Tukey's multiple comparisons test; ${ }^{\#} P<$ 0.01 and ${ }^{\S} P<0.05$ vs. the same time point of wild-type by Student's $t$ test. (F) $* P<$ 0.05 vs. own baseline, day 0 , by Tukey's multiple comparisons test; ${ }^{\#} P<0.01$ vs. the same time point of wild-type by Student's $t$ test. (G) $* P<0.05$ and $* * P<$ $0.01 \mathrm{vs}$. the same time point of wild-type by Student's $t$ test. $(\mathrm{H}) * P<0.05$ vs. day 21 of wild-type by Student's $t$ test. 
Expression of COX Isozymes and Prostanoid Synthases in DSS-Induced Colitis. To clarify the enzymatic pathway(s) involved in the augmented levels of $\mathrm{PGE}_{2}$ and $\mathrm{PGD}_{2}$ found in the inflamed colon of mice treated with DSS, we assessed the protein levels of COX-1 and COX-2 and major downstream synthases: mPGES1, hematopoietic HPGDS, and LPGDS. As shown in Supplemental Fig. 3, A-C, at baseline, COX-2 was almost undetectable, whereas COX-1 was expressed in the colonic mucosa. On day 5, COX-2 was dramatically upregulated and the levels returned to baseline values on day 21 (Supplemental Fig. 3, A and C). Similar timedependent changes were detected for mPGES1 expression (Supplemental Fig. 3, A and D). A significant increase in response to DSS was found for COX-1 and HPGDS expression, on days 5 and 21 (Supplemental Fig. 3, A, B, and E). Low levels of LPGDS were detected at each time point (Supplemental Fig. 3, A and F).

Overall, these results suggest the involvement of the COX-2/ mPGES1 pathway in enhanced biosynthesis of $\mathrm{PGE}_{2}$ in the inflamed colon of wild-type mice. COX-1 is likely to contribute to enhanced colonic $\mathrm{PGD}_{2}$ biosynthesis mainly through the coupling with HPGDS (Kämpfer et al., 2003).

In COX-1 cKO mice, a lower expression of COX-2, mPGES1, and HPGDS (Supplemental Fig. 3, A and C-E) was detected. These changes can contribute to the reduced capacity of colonic tissues to form $\mathrm{PGE}_{2}$ and $\mathrm{PGD}_{2}$, as compared with wild-type mice.

Colonic Levels of Cytokines in DSS-Induced Colitis and Effects of Platelet COX-1 Deletion. As shown in Fig. 3 , in wild-type mice on day 5 , the anti-inflammatory and immune-suppressive cytokines IL-10 and TGF- $\beta 1$ (Sanjabi et al., 2009) were significantly reduced in colonic tissues, whereas the proinflammatory IL-6 (Fernando et al., 2016) was significantly increased. On day 21 , IL-6 returned to baseline values (Fig. 3C).

In COX-1 cKO mice, on day 5 , the levels of the proinflammatory IL-6 were comparable to baseline, whereas the antiinflammatory IL-10 and TGF- $\beta 1$ levels resulted enhanced (Fig. 3).

The Impact of Platelet COX-1 Deletion on Histopathological Features of DSS-Induced Colitis. Sections of the distal colon were collected at baseline (day 0), day 5 (acute phase), and day 21 (chronic phase) and stained with $\mathrm{H} \& \mathrm{E}$ (Fig. 4A). As shown in Fig. 4B, necrotic areas were detected on day 5 of DSS administration in wild-type mice and persisted after removal of the chemical insult. In mice with specific deletion of platelet COX-1, the colonic necrotic areas were reduced, but not abrogated (Fig. 4B). The total histopathological scores [calculated as the sum of ulcer score, ulcer score area, inflammatory score, and inflammatory score area (Supplemental Fig. 4, A-D)] assessed in the colon sections of wild-type and COX-1 cKO mice are shown in Fig. 4C. Mice with platelet COX-1 deletion showed a statistically significant reduction in the histopathological scores (Fig. 4C; Supplemental Fig. 4, A-D).

We next performed immunofluorescent labeling-based image analysis to quantify the presence of macrophages (F4/80positive cells) and myofibroblasts (identified by staining for vimentin and $\alpha$-SMA), in the lamina propria of colon collected from mice with DSS-induced colitis on days 0,5 , and 21 (Fig. 4, $\mathrm{D}$ and E, respectively; Supplemental Fig. 5, A and B). The number of F4/80-positive cells was significantly $(P<0.05)$ lower in COX-1 cKO versus wild-type mice, on day 5 of DSS
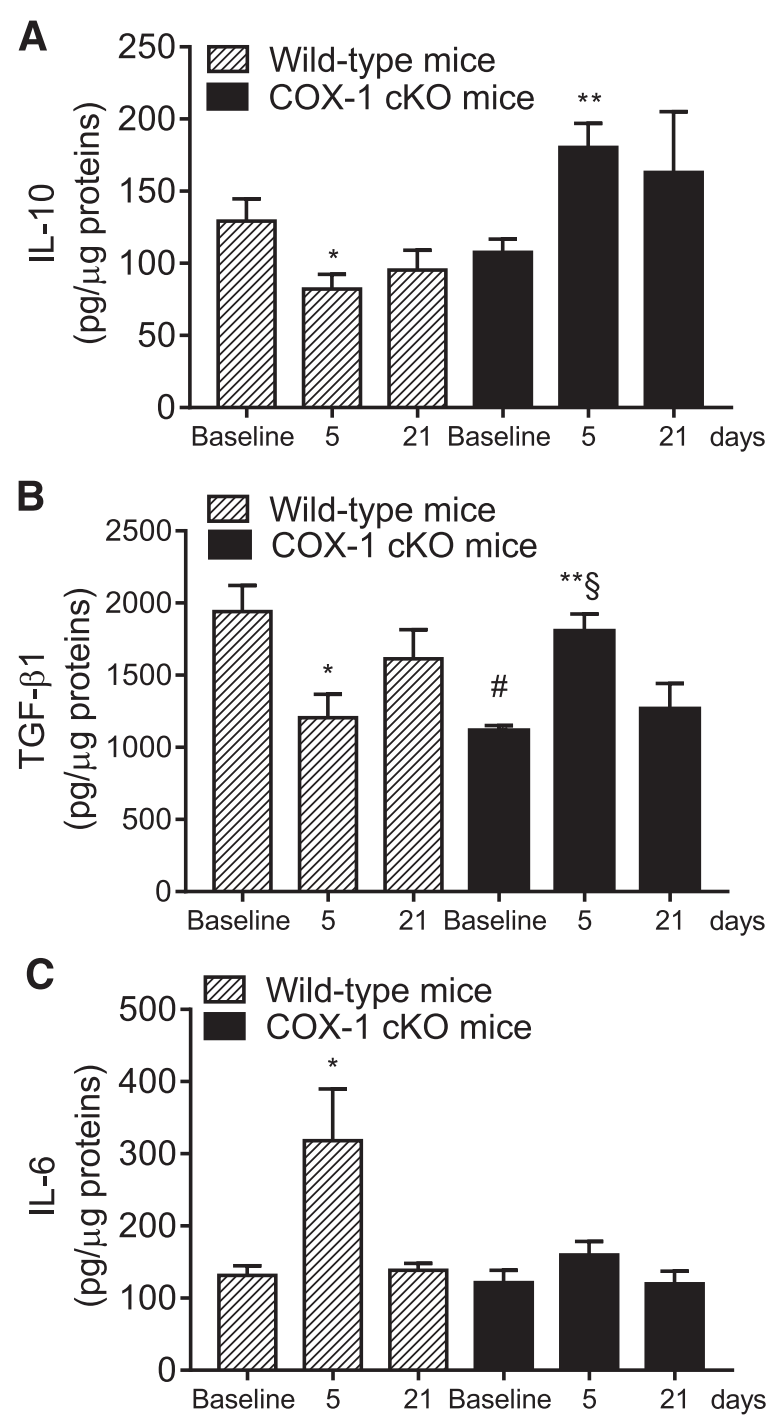

Fig. 3. Effects of platelet COX-1 deletion on colonic cytokine biosynthesis in mice treated with DSS. (A-C) In colonic tissues collected at baseline, day 0 , on day 5 of DSS administration (acute phase), and on day 21 after DSS removal (chronic phase) from wild-type and COX-1 cKO mice, the levels of IL-10, TGF- $\beta 1$, and IL- 6 were assessed by specific immunoassays; values were normalized for tissue protein concentration and are reported as picograms per microgram proteins, mean \pm S.E.M. $(n=3-7)$. (A) $* P<$ 0.05 vs. own baseline, day 0 , by Tukey's multiple comparisons test; ** $P<$ 0.01 vs. the same time point of wild-type by Student's $t$ test. (B) $* P<0.05$; $* * P<0.01$ vs. own baseline, day $0,{ }^{\S} P<0.05$ vs. day 21 of COX $-1 \mathrm{cKO}$ by Tukey's multiple comparisons test; ${ }^{\#} P<0.01$; ${ }^{\S} P<0.05$ vs. the same time point of wild-type by Student's $t$ test. (C) $* P<0.05$ vs. own baseline, day 0 , by Tukey's multiple comparisons test.

treatment (Fig. 4D; Supplemental Fig. 5A). Myofibroblast number was significantly enhanced on days 5 and 21 versus baseline, in wild-type mice. In COX-1 cKO mice, their number was increased on day 5 and returned to baseline values on day 21 , when it was significantly lower than in wild-type mice (Fig. 4E; Supplemental Fig. 5B).

Intestinal fibrosis was evaluated by Masson's trichrome staining (Fig. 4, F and G). The tissue area covered by collagen was measured with Image $J$ Software and expressed as the percentage of the entire cross-section area. In wild-type mice, collagen area was increased on day 21 versus baseline (Fig. 4 , $\mathrm{F}$ and $\mathrm{G})$ to a significantly $(P<0.01)$ greater extent than in COX-1 cKO mice (Fig. 4G). 


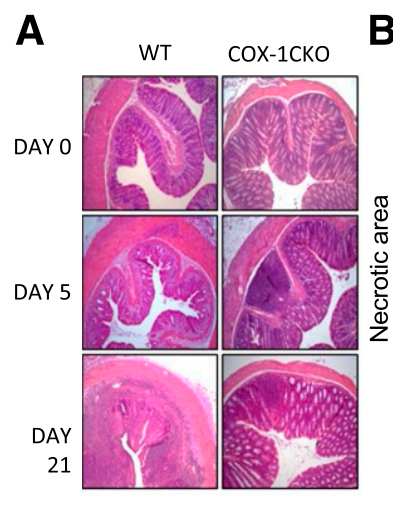

B

\section{C}
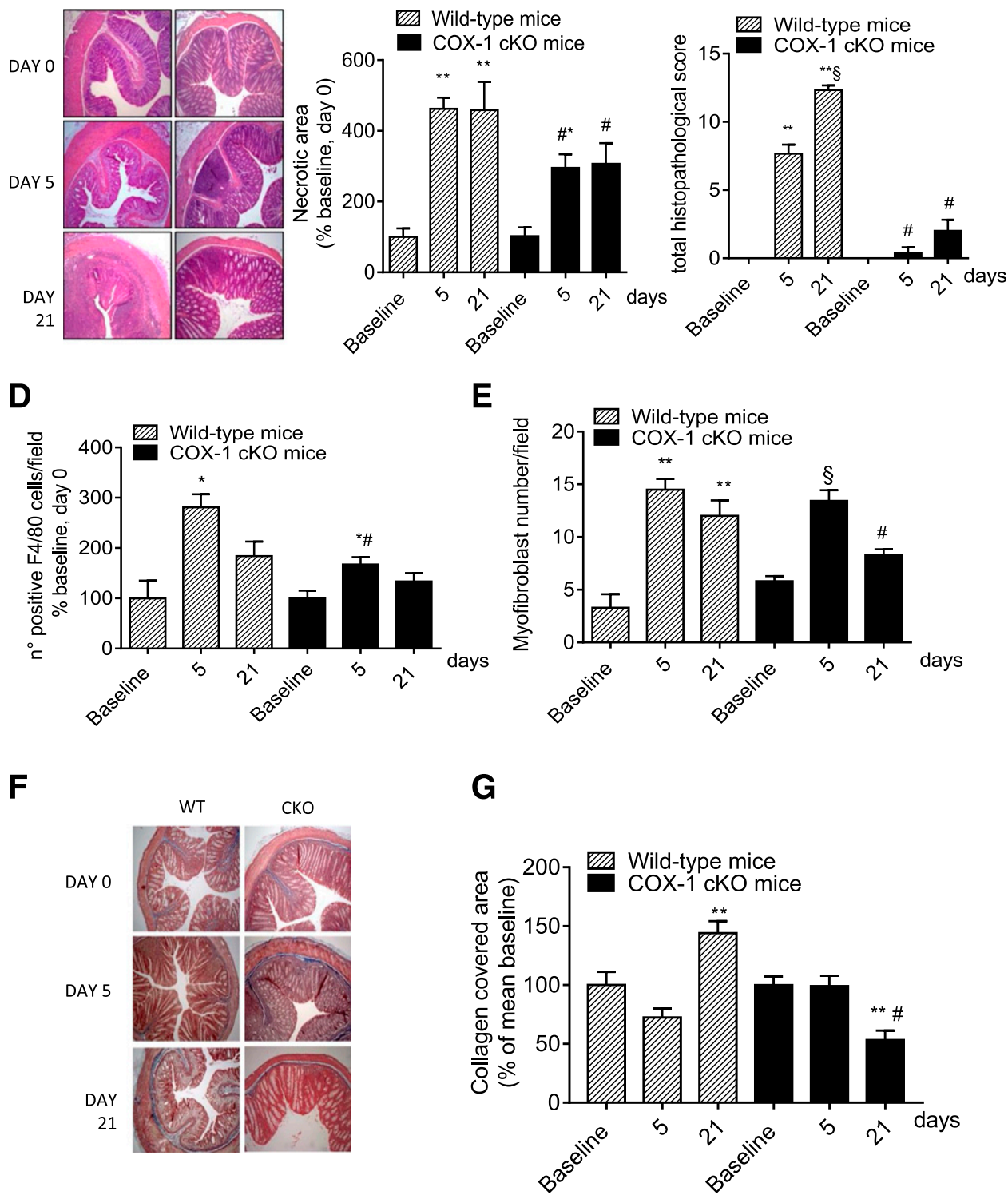

Fig. 4. The impact of platelet COX-1 deletion on histopathological features of DSS-induced colitis. C57BL/6 wild-type (WT) and COX-1 cKO mice were administered with $2 \%$ DSS for 5 days (acute phase), followed by administration of tap water for 16 days (chronic phase); distal colon samples were collected at baseline (day 0 ) and days 5 and 21, and sections were stained with H\&E for the evaluation of histopathological features. (A) Representative images of the histologic sections, Original magnification, 10×. (B) Necrosis areas were evaluated in H\&E sections and quantified using ImageJ software; necrosis areas were expressed as percentage of baseline, day 0 , data are reported as mean \pm S.E.M. $(n=3-5)$, ${ }^{* *} P<0.01$ vs. own baseline, day 0 , ${ }^{\#} P<$ 0.01 vs. own baseline, day 0 , by Tukey's multiple comparisons test; ${ }^{*} P<0.05$ vs. day 5 WT by Student's $t$ test. (C) Total histopathological score was calculated as the sum of ulcer score, ulcer score area, inflammatory score, and inflammatory score area in H\&E sections; data are reported as mean \pm S.E.M. $(n=3-5),{ }^{*} P<0.01$ vs. own baseline, day $0,{ }^{\S} P<0.01$ vs. day 5 wild-type by Tukey's multiple comparisons test; ${ }^{\#} P<0.01$ vs. the same time point of wild-type by Student's $t$ test. (D) Immunofluorescence analysis of macrophages (F4/80-positive cells) in the colon of DSS-induced colitis was assessed by confocal microscopy, on days 0,5 , and $21 ; \mathrm{F} 4 / 80$-positive cells/field were calculated, and data are reported as percentage of baseline, day 0 , mean \pm S.E.M. $(n=3-5)$; ${ }^{*} P<0.05$ vs. own baseline, day 0 , by Tukey's multiple comparisons test; ${ }^{\#} P<0.01$ vs. the same time point of wild-type by Student's $t$ test. (E) Immunofluorescence analysis of myofibroblasts in the colon of DSS-induced colitis on days 0,5 , and 21 ; myofibroblasts were identified by staining for vimentin and $\alpha$-SMA; data are reported as myofibroblasts/field, mean \pm S.E.M. $(n=3-5),{ }^{*} P<0.01$ vs. own baseline, day $0,{ }^{\S} P<0.01$ vs. own baseline, day 0 , and day 21 of COX-1 cKO by Tukey's multiple comparisons test; ${ }^{~} P<0.05$ vs. day 21 of wild-type by Student's $t$ test. (F and G) Intestinal fibrosis was evaluated by Masson's trichrome staining; the extracellular matrix is blue in color; the collagen-covered areas were measured by ImageJ Software and expressed as percentage of total area; data are reported as mean \pm S.E.M. Image numbers analyzed [day 0 (WT), 5 (WT), 21 (WT), day 0 (COX-1 cKO), $5(\mathrm{COX}-1 \mathrm{cKO})$, and $21(\mathrm{COX}-1 \mathrm{cKO})]: n=30,30,30,30,40$, and 40 , respectively. ** $P<0.01$ vs. own baseline, day 0 , and day 5 by Tukey's multiple comparisons test; ${ }^{\#} P<0.01$ vs. day 21 of wild-type by Student's $t$ test.

Overall, these results demonstrate a critical role of platelet COX-1 in the development of DSS-induced colitis symptoms, colonic histologic changes, and inflammation-associated fibrosis.

Effects of Platelets on Myofibroblast Function In Vitro. Platelets extravasated in the colonic lamina propria
(Fig. 5, A and B). Thus, we hypothesized that they contribute to the development of chronic inflammation-associated fibrosis through the crosstalk with intestinal myofibroblasts. To address this hypothesis, in vitro studies were performed using cocultures of human platelets and human 
intestinal myofibroblasts. At 24 hours of coculture with platelets, morphologic changes were observed in myofibroblasts (Fig. 5C). In the presence of platelets, the typical spindleshaped myofibroblast acquired a polarized phenotype manifested by an elongated cell body. These morphologic changes were prevented by the exposure of platelets to aspirin (an irreversible inhibitor of COX-1) (Simmons et al., 2004), which was then washed away before incubation with myofibroblasts (Fig. 5C). This finding suggests a platelet COX-1-dependent mechanism in the morphologic changes induced by platelets. $\mathrm{TXB}_{2}$ was the major product of arachidonic acid metabolism measured in the conditioned medium of plateletmyofibroblast coculture, and the levels were profoundly reduced in the presence of aspirinated platelets (Fig. 5D). Antagonism of TP receptor by SQ 29,548 also prevented the platelet-dependent induction of morphologic changes in the myofibroblasts (Fig. 5C). These morphologic changes were associated with reduced levels of $\alpha$-SMA, both as evaluated by confocal microscopy (Fig. 5, C and E) and mRNA (Fig. 5F). Both aspirin and the TP antagonist prevented downregulation of $\alpha$-SMA in myofibroblasts incubated with platelets (Fig. 5, C, E, and F). Using the TXA 2 mimetic U46619, we found that TP activation downregulated the expression of $\alpha$-SMA both by a direct effect and through the interference of TGF- $\beta 1$-dependent induction of $\alpha$-SMA (Fig. $5 \mathrm{G}$ ). TGF- $\beta 1$ was used at the concentration of $0.33 \mathrm{ng} / \mathrm{ml}$ comparable to that detected in the coculture of platelets and myofibroblasts (data not shown).

The myofibroblasts cultured with platelets were characterized by enhanced proliferative capacity (as assessed by confocal microscopy) (Fig. 5, H and I), which was prevented by the incubation with aspirinated platelets or with SQ 29,548, suggesting the role of platelet-derived $\mathrm{TXA}_{2}$ (Fig. 5, $\mathrm{H}$ and I).

Myofibroblasts cocultured with platelets were characterized by enhanced migratory ability (assessed using the scratch assay) (Fig. 6, A and B) and expression of RhoA (Fig. 6C), both of which were prevented by aspirin.

Finally, myofibroblasts cocultured with platelets were characterized by enhanced expression of mesenchymal markers, such as vimentin and fibronectin, and these effects were abrogated by the incubation of the myofibroblasts with aspirinated platelets (Fig. 6, D and E).

Thus, platelet-derived $\mathrm{TXA}_{2}$ may play an important role in the development of chronic inflammation and fibrosis by the induction of fibroblasts with proliferative, migratory, and mesenchymal features, which may promote a persistent proinflammatory state in the colonic mucosa.

\section{Discussion}

Patients with IBD are characterized by altered platelet functions (Kapsoritakis et al., 2001; Danese et al., 2004; Giannotta et al., 2015), which are reproduced in DSSinduced colonic inflammation in mice (Yan et al., 2013). To demonstrate the contribution of platelets to the development of inflammation and fibrosis of the gut in response to chemically-induced epithelial damage, we generated mice with a specific deletion of COX-1 in megakaryocytes/platelets, which were challenged with DSS. These mice showed a biochemical phenotype that recapitulates the selective inhibition of platelet COX-1 by low-dose aspirin in humans: virtually complete suppression of platelet $\mathrm{TXA}_{2}$ generation with substantial sparing of vascular prostacyclin biosynthesis (Capone et al., 2004).

The capacity of the colon to generate $\mathrm{TXA}_{2}$ was not significantly altered in the acute and the recovery phase of DSS-induced colitis. However, enhanced systemic biosynthesis of $\mathrm{TXA}_{2}$, as assessed by measuring the urinary levels of TX-M (FitzGerald et al., 1983), was detected reaching a significant difference versus baseline in the chronic stage of the disease. These findings suggest the occurrence of systemic activation of platelets in colitis. In fact, the assessment of urinary TX-M is considered a noninvasive marker of in vivo platelet activation (FitzGerald et al., 1983; Grosser et al., 2018). These results suggest that platelet-derived $\mathrm{TXA}_{2}$ played a crucial role in the development of colitis. Colonic $\mathrm{TXA}_{2}$ can derive from various cell types, including epithelial cells, inflammatory cells, and platelets. The assessment of the colonic capacity to produce $\mathrm{TXA}_{2}$ in tissue homogenates ex vivo is not appropriate to detect the actual contribution of the different cell types in vivo, thus explaining the fact that specific deletion of platelet COX-1 was not associated with a significant reduction in colonic $\mathrm{TXB}_{2}$ levels.

Platelets are activated immediately in response to tissue damage, and may, in turn, trigger an inflammatory response. This occurs via the platelet release into the extracellular environment of different soluble mediators (both lipids, such as $\mathrm{TXA}_{2}$ and proteins) and vesicles rich in genetic material (e.g., microRNAs), which contribute to wound repair (Gawaz et al., 2005; Dovizio et al., 2017). It is a phenomenon requiring the time- and space-controlled induction of cellular migration, extracellular matrix organization/remodeling, cell proliferation, differentiation, and angiogenesis/neovascularization (Landén et al., 2016). However, under pathologic conditions, including atherothrombosis (Davì and Patrono, 2007), colorectal cancer (Patrignani and Patrono, 2016), and-as shown in this work-intestinal colitis, the occurrence of an uncontrolled platelet activation can contribute to the development of a chronic inflammatory state that favors the disease progression. Among the soluble mediators released from activated platelets, we show that enhanced $\mathrm{TXA}_{2}$ production plays a crucial role in the pathogenesis of intestinal colitis. TXA $_{2}$ is known to increase microvascular permeability (Turnage et al., 1997) and orchestrate the trafficking of inflammatory and immune cells and platelets and their accumulation in the colon (Vitiello et al., 2014). In colitis, platelets extravasated into the colonic interstitium (Danese et al., 2004; Petito et al., 2017) may promote the development of chronic inflammationassociated fibrosis through the crosstalk with myofibroblasts. In vitro, we found that platelet-derived $\mathrm{TXA}_{2}$ induced phenotypic and functional changes of myofibroblasts such as the reduction in $\alpha$-SMA and the increase in vimentin, fibronectin, and RhoA expression associated with an enhanced capacity to proliferate and migrate. These effects were prevented by inhibition of platelet COX-1 by aspirin or by TP antagonism. It has been previously shown that $\alpha$-SMA deficiency in myofibroblasts promotes renal fibrosis (Takeji et al., 2006). The cross-talk of platelets with stromal cells in the gut may also contribute to the development of colorectal cancer by enhancing the release of growth factors and lipid mediators, including prostanoids, which induce a malignant program in epithelial cells (Dovizio et al., 2017). 
A

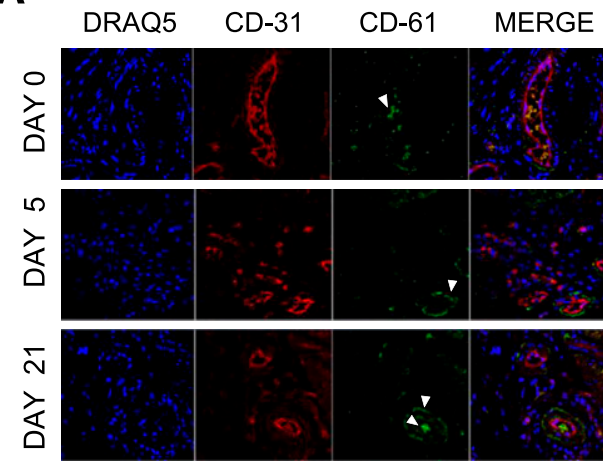

C

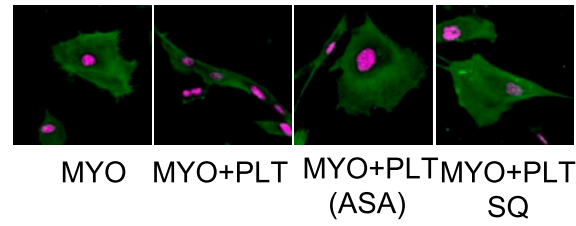

E

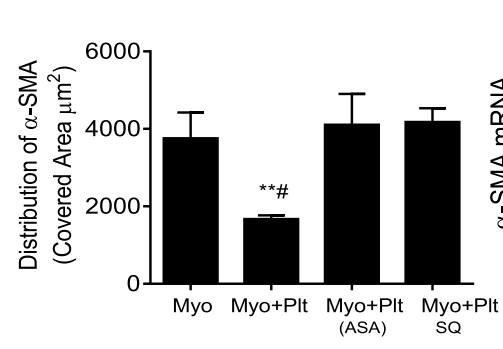

H

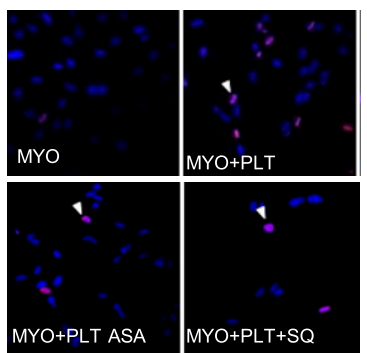

F

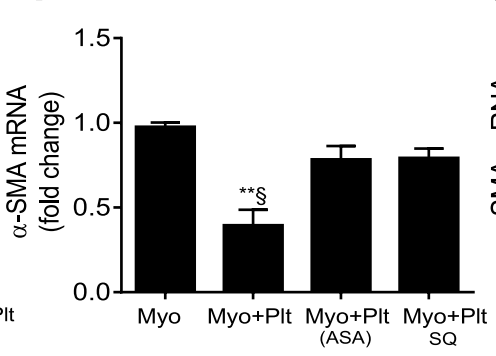

I

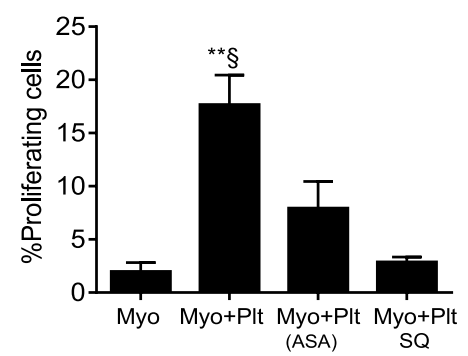

G
B
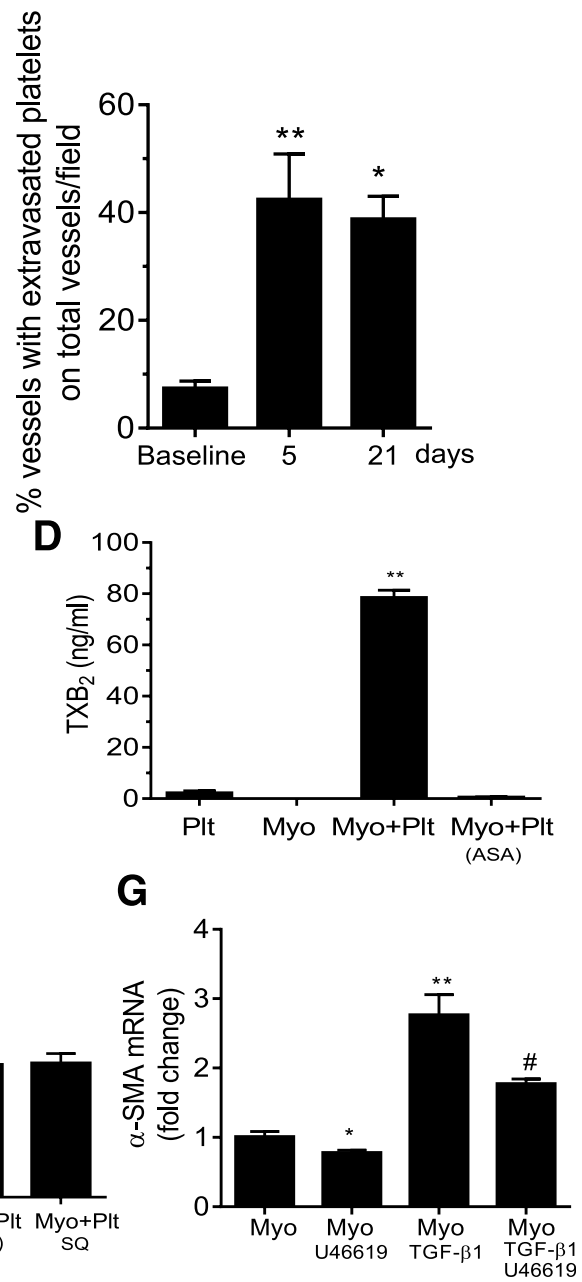

Fig. 5. Effects of platelets on myofibroblast function. (A and B) DSS colitis was induced in wild-type mice, and colon samples were evaluated for platelet extravasation on days 0,5 , and 21 by immunofluorescence using confocal microscopy; a representative immunofluorescence staining of colon sections is shown [CD31 (red), an endothelial marker, CD61 (green), a platelet marker, and 1,5-bis\{[ 2-(dimethylamino) ethyl] amino\}-4,8-dihydroxyanthracene9,10-dione (blue), a nuclear marker] (A); vessels with extravasated platelets were counted and reported as percentage of total vessels per field (B), mean \pm S.E.M. $(n=3), * P<0.05$ and $* * P<0.01$ vs. baseline, day 0 , by Tukey's multiple comparisons test. (C-F) Human intestinal myofibroblast cells $\left(9 \times 10^{4}\right)\left(\right.$ Myo) were cultured alone or cocultured with isolated human platelets (Plt, $\left.0.5 \times 10^{8}\right)$ (Myo + Plt) for 24 hours, untreated or pretreated with aspirin (ASA, acetylsalicylic acid) $100 \mu \mathrm{M}$; in some experiments, Myo were incubated with Plt, in the presence of DMSO vehicle or the TP receptor antagonist SQ 29,548 (SQ, $10 \mu \mathrm{M}$ ); (C) representative image of immunofluorescence analysis of $\alpha$-SMA (in green) by confocal microscopy; (D) TXB levels $^{-}$ assessed by immunoassay, mean \pm S.E.M. $(n=4-13)$, **P<0.01 vs. the other conditions by Tukey's multiple comparisons test; (E) quantification of $\alpha$-SMA-covered area using confocal microscopy, in myofibroblasts, mean \pm S.E.M. $(n=5-15),{ }^{\#} P<0.05$ vs. Myo and Myo + Plt (ASA) and ** $P<0.01$ vs. Myo + Plt + SQ by Tukey's multiple comparisons test. (F) $\alpha$-SMA mRNA levels by quantitative real-time PCR, normalized to glyceraldehyde-3phosphate dehydrogenase mRNA levels, reported as fold-change vs. myofibroblast alone (Myo), mean \pm S.E.M. $(n=3-7)$, ${ }^{* *} P<0.01$ vs. Myo and Myo + Plt (ASA), ${ }^{\S} \mathrm{P}<0.05$ vs. Myo + Plt + SQ by Tukey's multiple comparisons test. (G) mRNA levels of $\alpha$-SMA/glyceraldehyde-3-phosphate dehydrogenase by quantitative PCR in Myo treated with DMSO, U46619 (250 nM, a TXA 2 mimetic), TGF- $\beta 1$ (0.33 ng/ml), or TGF- $\beta 1-\mathrm{U} 46619$, for 48 hours; data are reported as fold-change vs. Myo alone, mean \pm S.E.M. $(n=3-5), * * P<0.01$ vs. Myo and Myo $+\mathrm{U} 46619, * P<0.05$ vs. Myo + TGF- $\beta 1+\mathrm{U} 46619$, ${ }^{*} P<$ 0.05 vs. Myo + TGF- $\beta 1$ by Tukey's multiple comparisons test. (H and I) Under the same experimental conditions described above (C-F), cell proliferation was evaluated by confocal microscopy (Click-iT EdU assay), and a representative immunofluorescence staining of Myo (in blue, cell nuclei; in pink, DNAincorporating thymidine) is shown (H); percentage of proliferating cells (pink) vs. total cells is shown (I), mean \pm S.E.M. $(n=4-8)$, $* * P<0.01$ vs. Myo and Myo + Plt $+\mathrm{SQ},{ }^{\S} \mathrm{P}<0.05$ vs. Myo + Plt (ASA) by Tukey's multiple comparisons test. 
A

B

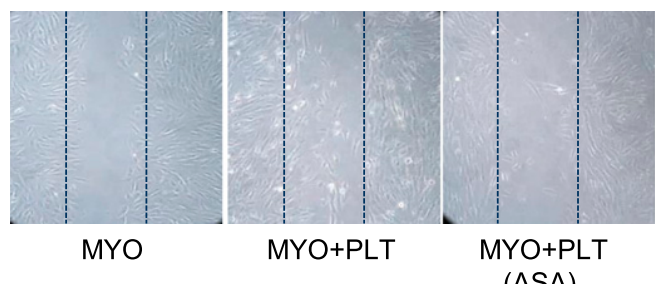

(ASA)

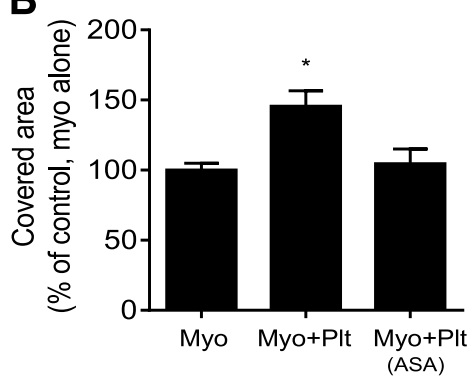

$\mathbf{E}$

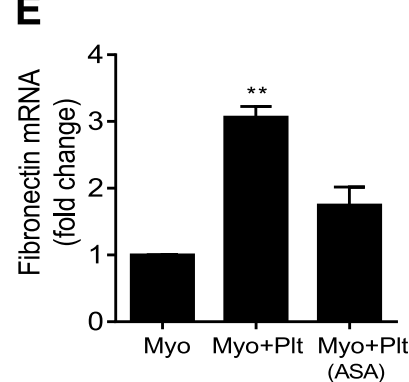

Fig. 6. Effects of platelets on myofibroblast migration and gene expression. Myofibroblast cells (Myo, $9 \times 10^{4}$ ) were cultured alone or cocultured with platelets (Plt, $0.5 \times 10^{8}$ ), untreated or pretreated with aspirin (ASA, acetylsalicylic acid, $100 \mu \mathrm{M}$ ), for 24 hours. (A) Cell migration assay was performed using an in vitro scratch assay, and representative phase-contrast images are shown. (B) The percentage of the covered area was measured using Image $\mathrm{J}$ software, and data are reported as percentage of control (myofibroblasts cultured alone, Myo) (mean \pm S.E.M., $n=4$ ); ${ }^{*} P<0.05$ vs. Myo and Myo + Plt (ASA) by Tukey's multiple comparisons test. In the same experimental conditions, Myo were assessed for mRNA levels of RhoA (C), vimentin (D), and fibronectin (E) by quantitative real-time PCR and normalized to glyceraldehyde-3-phosphate dehydrogenase mRNA levels; values are reported as foldchange vs. Myo cultured alone (mean \pm S.E.M., $n=3-8$ ), ${ }^{*} * P<0.01$ vs. Myo and ${ }^{\S} P<0.05$ vs. Myo + Plt (ASA) by Tukey's multiple comparisons test (C), ** $P<0.01$ vs. Myo and Myo + Plt (ASA) by Tukey's multiple comparisons test (D and E).

Although the DSS-induced colitis model has some similarities with UC and has been extensively used due to its rapidity, simplicity, and reproducibility (Eichele and Kharbanda, 2017), it has some limitations, including the fact that, unlike the human disease, $\mathrm{T}$ and $\mathrm{B}$ cells are not required for the development of colitis (Chassaing et al., 2014). However, it is noteworthy that no available animal model may serve as a completely valid surrogate for the human disease (Mizoguchi and Mizoguchi, 2010).

The strength of the present study is that we generated mice with specific deletion of COX-1 in platelets that allowed us to identify $\mathrm{TXA}_{2}$-dependent platelet activation as an important source of mediators contributing to intestinal inflammation and fibrosis. Moreover, this unique mouse model provides a novel tool to investigate the involvement of platelets in several pathologies, including colorectal cancer.

Limited clinical data are available on the effect of antiplatelet agents in IBD. No randomized clinical studies have been performed with low-dose aspirin. The results of epidemiologic studies with aspirin showed conflicting results, and no information on the effect of aspirin at low doses is available (Maaser et al., 2017).

In conclusion, our results highlight the role of plateletderived $\mathrm{TXA}_{2}$ in the development of colitis and fibrosis induced by epithelial damage. The findings of the present study provide the rationale to investigate the potential efficacy and safety of low-dose aspirin in limiting the inflammation and tissue damage associated with IBD. This antiplatelet agent should not aggravate the intestinal bleeding because clinical data show that low-dose aspirin causes bleeding, mainly from pre-existing lesions, in the stomach and duodenum (Sostres and Lanas, 2011), which are sites not involved in UC and very rarely involved in Crohn's disease.
This is also supported by our findings showing that, in DSSinduced colitis, deletion of COX-1 in platelets was not associated with any significant change in EMO score versus wild-type mice. Low-dose aspirin, through downstream effects of platelet inhibition, might also reduce the enhanced risk of colon cancer in IBD patients (Patrignani and Patrono, 2016).

\section{Acknowledgments}

We thank the undergraduate student Lorenzo Fiorino, "G. d'Annunzio" University, for enthusiasm, dedication, and exceptional technical assistance.

\section{Authorship Contributions}

Participated in research design: Patrignani, Sacco, Bruno, Contursi, Dovizio.

Conducted experiments: Sacco, Bruno, Tacconelli, Contursi, Dovizio, Ricciotti, Guillem-Llobat, Salvatore, Di Francesco, Fullone, Alberti, Arena, Liu, Gong.

Contributed new reagents or analytic tools: $\mathrm{Yu}$.

Performed data analysis: Sgambato, Ballerini, Patrignani.

Wrote or contributed to the writing of the manuscript: Patrignani, FitzGerald, Patrono, Yu.

\section{References}

Abraham $\mathrm{C}$ and Cho JH (2009) Inflammatory bowel disease. $N$ Engl J Med 361: 2066-2078

Ananthakrishnan AN (2015) Epidemiology and risk factors for IBD. Nat Rev Gastroenterol Hepatol 12:205-217.

Boulet L, Ouellet M, Bateman KP, Ethier D, Percival MD, Riendeau D, Mancini JA and Méthot N (2004) Deletion of microsomal prostaglandin E2 (PGE2) synthase-1 reduces inducible and basal $\mathrm{PGE}_{2}$ production and alters the gastric prostanoid profile. J Biol Chem 279:23229-23237.

Bruggeman LA, Horigan EA, Horikoshi S, Ray PE, and Klotman PE (1991) Thromboxane stimulates synthesis of extracellular matrix proteins in vitro. Am J Physiol 261:F488-F494.

Capone ML, Tacconelli S, Sciulli MG, Grana M, Ricciotti E, Minuz P, Di Gregorio P, Merciaro G, Patrono C, and Patrignani P (2004) Clinical pharmacology of platelet, monocyte, and vascular cyclooxygenase inhibition by naproxen and low-dose aspirin in healthy subjects. Circulation 109:1468-1471. 
Carter PR, McElhatten RM, Zhang S, Wright WS, and Harris NR (2011) Thromboxane-prostanoid receptor expression and antagonism in dextran-sodium sulfate-induced colitis. Inflamm Res 60:87-92.

Ceuppens JL, Vertessen S, Deckmyn H, and Vermylen J (1985) Effects of thromboxane A2 on lymphocyte proliferation. Cell Immunol 90:458-463.

Chassaing B, Aitken JD, Malleshappa M, and Vijay-Kumar M (2014) Dextran sulfate sodium (DSS)-induced colitis in mice. Curr Protoc Immunol 104:Unit 15.25.

Choi CR, Bakir IA, Hart AL, and Graham TA (2017) Clonal evolution of colorectal cancer in IBD. Nat Rev Gastroenterol Hepatol 14:218-229.

Cooper HS, Murthy SN, Shah RS, and Sedergran DJ (1993) Clinicopathologic study of dextran sulfate sodium experimental murine colitis. Lab Invest 69:238-249.

Danese S, Motte Cd C, and Fiocchi C (2004) Platelets in inflammatory bowel disease: clinical, pathogenic, and therapeutic implications. Am J Gastroenterol 99:938-945. Davì G and Patrono C (2007) Platelet activation and atherothrombosis. N Engl J Med 357:2482-2494.

Di Francesco L, Totani L, Dovizio M, Piccoli A, Di Francesco A, Salvatore T, Pandolfi A, Evangelista V, Dercho RA, Seta F, et al. (2009) Induction of prostacyclin by steady laminar shear stress suppresses tumor necrosis factor-alpha biosynthesis via heme oxygenase-1 in human endothelial cells. Circ Res 104:506-513.

Din FV, Valanciute A, Houde VP, Zibrova D, Green KA, Sakamoto K, Alessi DR, and Dunlop MG (2012) Aspirin inhibits mTOR signaling, activates AMP-activated protein kinase, and induces autophagy in colorectal cancer cells. Gastroenterology 142:1504-1515.e3.

Dixon DA, Tolley ND, Bemis-Standoli K, Martinez ML, Weyrich AS, Morrow JD, Prescott SM, and Zimmerman GA (2006) Expression of COX-2 in platelet-monocyte interactions occurs via combinatorial regulation involving adhesion and cytokine signaling. J Clin Invest 116:2727-2738.

Dovizio M, Alberti S, Guillem-Llobat P, and Patrignani P (2014) Role of platelets in inflammation and cancer: novel therapeutic strategies. Basic Clin Pharmacol Toxicol 114:118-127.

Dovizio M, Sacco A, and Patrignani P (2017) Curbing tumorigenesis and malignant progression through the pharmacological control of the wound healing process. Vascul Pharmacol 89:1-11.

Eichele DD and Kharbanda KK (2017) Dextran sodium sulfate colitis murine model: an indispensable tool for advancing our understanding of inflammatory bowel diseases pathogenesis. World J Gastroenterol 23:6016-6029.

Fernando MR, Giembycz MA, and McKay DM (2016) Bidirectional crosstalk via IL-6, PGE2 and PGD2 between murine myofibroblasts and alternatively activated macrophages enhances anti-inflammatory phenotype in both cells. $\mathrm{Br}$ J Pharmacol 173:899-912.

FitzGerald GA, Pedersen AK, and Patrono C (1983) Analysis of prostacyclin and thromboxane biosynthesis in cardiovascular disease. Circulation 67:1174-1177.

Gawaz M, Langer H, and May AE (2005) Platelets in inflammation and atherogenesis. J Clin Invest 115:3378-3384.

Giannotta M, Tapete G, Emmi G, Silvestri E, and Milla M (2015) Thrombosis in inflammatory bowel diseases: what's the link? Thromb $J$ 13:14

Grosser T, Naji A, and FitzGerald GA (2018) Urinary prostaglandin metabolites: an incomplete reckoning and a flush to judgment. Circ Res 122:537-539.

Guillem-Llobat P, Dovizio M, Bruno A, Ricciotti E, Cufino V, Sacco A, Grande R, Alberti S, Arena V, Cirillo M, et al. (2016) Aspirin prevents colorectal cancer metastasis in mice by splitting the crosstalk between platelets and tumor cells. Oncotarget 7:32462-32477.

Hawkey CJ, Karmeli F, and Rachmilewitz D (1983) Imbalance of prostacyclin and thromboxane synthesis in Crohn's disease. Gut 24:881-885.

Joung JK and Sander JD (2013) TALENs: a widely applicable technology for targeted genome editing. Nat Rev Mol Cell Biol 14:49-55.

Kämpfer H, Bräutigam L, Geisslinger G, Pfeilschifter J, and Frank S (2003) Cyclooxygenase-1-coupled prostaglandin biosynthesis constitutes an essential prerequisite for skin repair. J Invest Dermatol 120:880-890.

Kapsoritakis AN, Koukourakis MI, Sfiridaki A, Potamianos SP, Kosmadaki MG, Koutroubakis IE, and Kouroumalis EA (2001) Mean platelet volume: a useful marker of inflammatory bowel disease activity. Am J Gastroenterol 96:776-781.

Landén NX, Li D, and Ståhle M (2016) Transition from inflammation to proliferation: a critical step during wound healing. Cell Mol Life Sci 73:3861-3885.

Leung KH and Mihich E (1980) Prostaglandin modulation of development of cellmediated immunity in culture. Nature 288:597-600

Maaser C, Langholz E, Gordon H, Burisch J, Ellul P, Ramirez VH, Karakan T, Katsanos KH, Krustins E, Levine A, et al. (2017) European Crohn's and colitis organization topical review on environmental factors in IBD. J Crohn's Colitis 11: 905-920.

Mizoguchi A and Mizoguchi E (2010) Animal models of IBD: linkage to human disease. Curr Opin Pharmacol 10:578-587.

Okayasu I, Hatakeyama S, Yamada M, Ohkusa T, Inagaki Y, and Nakaya R (1990) A novel method in the induction of reliable experimental acute and chronic ulcerative colitis in mice. Gastroenterology 98:694-702.
Patrignani P and Patrono C (2016) Aspirin and cancer. J Am Coll Cardiol 68: 967-976.

Patrignani P, Sacco A, Sostres C, Bruno A, Dovizio M, Piazuelo E, Di Francesco L, Contursi A, Zucchelli M, Schiavone S, et al. (2017) Low-dose aspirin acetylates cyclooxygenase-1 in human colorectal mucosa: implications for the chemoprevention of colorectal cancer. Clin Pharmacol Ther 102:52-61.

Patrono C, Ciabattoni G, Pinca E, Pugliese F, Castrucci G, De Salvo A, Satta MA and Peskar BA (1980) Low dose aspirin and inhibition of thromboxane B2 production in healthy subjects. Thromb Res 17:317-327.

Petito E, Momi S, and Gresele P (2017) The migration of platelets and their interaction with other migrating cells, in Platelets in Thrombotic and NonThrombotic Disorders (Gresele P, Kleiman N, Lopez J, and Page C eds) pp 337-351, Springer, Cham, Switzerland.

Ray MK, Fagan SP, and Brunicardi FC (2000) The Cre-loxP system: a versatile too for targeting genes in a cell- and stage-specific manner. Cell Transplant 9:805-815.

Ricciotti E and FitzGerald GA (2011) Prostaglandins and inflammation. Arterioscler Thromb Vasc Biol 31:986-1000.

Rieder F and Fiocchi C (2008) Intestinal fibrosis in inflammatory bowel disease: current knowledge and future perspectives. J Crohn's Colitis 2:279-290.

Sanjabi S, Zenewicz LA, Kamanaka M, and Flavell RA (2009) Anti-inflammatory and pro-inflammatory roles of TGF-beta, IL-10, and IL-22 in immunity and autoimmunity. Curr Opin Pharmacol 9:447-453.

Shah B and Mayer L (2010) Current status of monoclonal antibody therapy for the treatment of inflammatory bowel disease. Expert Rev Clin Immunol 6: $607-620$

Simmons DL, Botting RM, and Hla T (2004) Cyclooxygenase isozymes: the biology of prostaglandin synthesis and inhibition. Pharmacol Rev 56:387-437.

Smyth EM, Grosser T, Wang M, Yu Y, and FitzGerald GA (2009) Prostanoids in health and disease. J Lipid Res 50 (Suppl):S423-S428.

Song WL, Lawson JA, Wang M, Zou H, and FitzGerald GA (2007) Noninvasive assessment of the role of cyclooxygenases in cardiovascular health: a detailed HPLC/ MS/MS method. Methods Enzymol 433:51-72.

Song WL, Wang M, Ricciotti E, Fries S, Yu Y, Grosser T, Reilly M, Lawson JA and FitzGerald GA (2008) Tetranor PGDM, an abundant urinary metabolite reflects biosynthesis of prostaglandin D2 in mice and humans. J Biol Chem 283: $1179-1188$

Sostres C and Lanas A (2011) Gastrointestinal effects of aspirin. Nat Rev Gastroenterol Hepatol 8:385-394.

Takeji M, Moriyama T, Oseto S, Kawada N, Hori M, Imai E, and Miwa T (2006) Smooth muscle alpha-actin deficiency in myofibroblasts leads to enhanced renal tissue fibrosis. J Biol Chem 281:40193-40200.

Terzuoli E, Corti F, Nannelli G, Giachetti A, Donnini S, and Ziche M (2018) Bradykinin B2 receptor contributes to inflammatory responses in human endothelial cells by the transactivation of the fibroblast growth factor receptor FGFR-1. Int J Mol Sci 19:1-16.

Thomas DW, Rocha PN, Nataraj C, Robinson LA, Spurney RF, Koller BH, and Coffman TM (2003) Proinflammatory actions of thromboxane receptors to enhance cellular immune responses. J Immunol 171:6389-6395.

Turnage RH, LaNoue JL, Kadesky KM, Meng Y, and Myers SI (1997) Thromboxane A2 mediates increased pulmonary microvascular permeability after intestinal reperfusion. J Appl Physiol (1985) 82:592-598.

Vitiello L, Spoletini I, Gorini S, Pontecorvo L, Ferrari D, Ferraro E, Stabile E, Caprio M, and La Sala A (2014) Microvascular inflammation in atherosclerosis. IJC Metab Endocr 3:1-7.

Wang D and DuBois RN (2013) Urinary PGE-M: a promising cancer biomarker. Cancer Prev Res (Phila) 6:507-510.

Weissmüller T, Campbell EL, Rosenberger P, Scully M, Beck PL, Furuta GT, and Colgan SP (2008) PMNs facilitate translocation of platelets across human and mouse epithelium and together alter fluid homeostasis via epithelial cell-expressed ecto-NTPDases. J Clin Invest 118:3682-3692.

Whittle BJ, Kauffman GL, and Moncada S (1981) Vasoconstriction with thromboxane A2 induces ulceration of the gastric mucosa. Nature 292:472-474.

Yan SL, Russell J, Harris NR, Senchenkova EY, Yildirim A, and Granger DN (2013) Platelet abnormalities during colonic inflammation. Inflamm Bowel Dis 19 $1245-1253$

Yu Y, Cheng Y, Fan J, Chen XS, Klein-Szanto A, Fitzgerald GA, and Funk CD (2005) Differential impact of prostaglandin $\mathrm{H}$ synthase 1 knockdown on platelets and parturition. J Clin Invest 115:986-995.

Address correspondence to: Dr. Paola Patrignani, Department of Neuroscience, Imaging, and Clinical Sciences, and CeSI-MeT, "G. d'Annunzio" University, Via dei Vestini 31,66100 Chieti, Italy. E-mail: ppatrignani@ unich.it 